\title{
Insecticidas botánicos registrados y no registrados en Nicaragua
}

\author{
Registered and unregistered botanical insecticides in Nicaragua
}

\author{
Edgardo Jiménez-Martínez ${ }^{1}$ \\ Ramiro Manzanares Rugama ${ }^{2}$
}

\section{Resumen}

En Nicaragua ha surgido la necesidad de profundizar en el análisis de alternativas naturales para el control de plagas en los cultivos, opción efectiva a los pesticidas sintéticos. Por esta razón es necesario pensar en nuevas alternativas de control de plagas que sean más amigable con el medio ambiente y que sean útiles en el manejo integrado de plagas, esta investigación fue cualitativa no experimental fundamentada en el método descriptivo de investigación aplicada en donde no hay manipulación de variables, analizando la realidad o situación determinada con una riqueza interpretativa, como parte de este proceso se realizó la recopilación, sistematización con un análisis de los principales insecticidas botánicos registrados y evaluados en Nicaragua, tomando como base las pruebas de efectividad que se utilizaron para registrar los productos en el Instituto de Protección y Sanidad Agropecuaria, el propósito de este estudio fue documentar la información existente sobre el uso de insecticidas botánicos registrados y no registrados en Nicaragua, en este estudio se encontró que en Nicaragua se han registrado 12 insecticidas botánicos desde el año 2011 hasta el año 2017, todos estos a base de extractos acuoso de planta, semillas, hojas y flores que se han utilizado en los cultivos de hortalizas, frutales, okra, raíces y tubérculos, para el control de plagas como mosca blanca, áfidos, ácaros y trips, también se encontró que en Nicaragua se han realizado ocho investigaciones en el periodo comprendido entre mayo 1990 y noviembre 2017 sobre el uso y manejo de insecticidas botánicos a base de extractos de chile, ajo, crisantemo, madero negro y Neem en los cultivos de tomate, chiltoma, repollo, sorgo y marañón con el propósito de controlar las principales plagas que atacan a estos cultivos.

Palabras clave: Insecticidas botánicos; Registro de insecticidas.

\section{Abstract}

In Nicaragua, it has arisen the need to deepen in the analysis of natural alternatives for the control of pests in crops, drawing attention to the application of various products of natural origin as an effective option to synthetic pesticides. For this reason, it is necessary to think of new alternatives for pest control that are more friendly to the environment and that are useful in the integrated management of pests, for this study, it was used a qualitative non-experimental research based on the descriptive method of applied research where there is no manipulation of variables, analyzing the reality or situation determined with an interpretative richness, as part of this process the compilation, systematization, with an analysis of the main botanical insecticides registered and evaluated in Nicaragua was carried out, based on the effectiveness tests that were used to register the products in the Agricultural Protection and Health Institute, the purpose of this study was to record the existing information on the use of registered and unregistered botanical insecticides in Nicaragua, in this study it was found that in Nicaragua, there has been registered 12 botanical insecticides from 2011 to 2017, all these based on aqueous extracts of plants, seeds, leaves and flowers that have been used in the crops of vegetables, fruit trees, okra, roots and tubers, for the pests control such as white flies, aphids, mites and thrips, it was also found that in Nicaragua eight investigations have been carried out in the period between May 1990 to November 2017 on the use and management of

\footnotetext{
1 PhD en Entomología, Docente Investigador, Director de Investigación, Extensión y Posgrado, Universidad Nacional Agraria, Nicaragua. Correo: edgardo.jimenez@ci.una.edu.ni https://orcid.org/0000-0003-1086-7380

2 MSc en Gestión de Medidas Sanitarias y Fitosanitarias. Instituto de Protección y Sanidad Agropecuaria.
}

Recibido: 03/o9/2020 - Aprobado: 14/10/2020 
botanical insecticides based on extracts of chili, garlic, chrysanthemum, black wood and Neem in tomato, chili peper, cabbage, sorghum and cashew crops in order to control the main pests that attack these crops.

Keywords: Botanical insecticides; Insecticide registration

\section{Introducción}

Los plaguicidas botánicos son derivados de algunas partes o ingredientes activos de las plantas. En los últimos años, la aplicación de varios productos de plantas medicinales ha llamado mucho la atención como alternativas efectivas a los pesticidas sintéticos, estos productos vegetales son muy eficaces, menos costosos, biodegradables y más seguros que sus equivalentes sintéticos, los cuales son altamente persistentes en el medio ambiente y tóxico para los organismos blandos, incluidos los humanos, a los cuales le causan muchas de las enfermedades no identificadas después de la bioacumulación (Singh et al., 1996).

El efecto nocivo de los extractos de plantas o sus compuestos puros contra los insectos se puede manifestar de diversas maneras, incluyendo la toxicidad, la mortalidad, inhibiendo el crecimiento, supresión de comportamiento reproductivo reduciendo la fertilidad y la fecundidad (Ben, 2001).

Se sabe que las plagas son responsables de grandes pérdidas anuales en la producción de los cultivos agrícolas y forestales, como parte de su control y a pesar del gran impacto de los plaguicidas en el avance de la agricultura moderna, el uso irracional de los compuestos orgánicos sintético ha provocado serios problemas globales, como la contaminación del medio ambiente, la acumulación de residuos tóxicos en los alimentos con perjuicios a la salud humana y animal, efectos negativos sobre insectos benéficos y la resistencia por parte de los organismos nocivos (Carrero, 1996).

Por esta razón es necesario pensar en nuevas alternativas de control de plagas que sean más amigable con el medio ambiente, como el uso de plantas dotadas de mecanismos para defenderse de sus enemigos. Esta problemática ha impulsado el uso de formulaciones de plantas con propiedades insecticidas y fungicidas, que permiten manejar las plagas, proteger el cultivo y obtener mayor rendimiento y calidad en la producción sin poner en riesgo la salud del hombre y su entorno (Rodríguez, 200o). Los extractos de origen vegetal han sido usados como productos insecticidas desde la antigüedad, en muchas regiones del mundo, especialmente en las comunidades indígenas donde se produce para autoconsumo, esta práctica se ha seguido usando a través de generaciones y representan un recurso renovable, más accesible y económico que los insecticidas químicos sintéticos (Alonso, 1998).

Se hace imprescindible el estudio de nuevas vías para el Manejo Integrado de Plagas (MIP), que es una metodología que emplea todos los procedimientos aceptables desde el punto de vista económico, ecológico y toxicológico para mantener las poblaciones de organismos nocivos por debajo del umbral económico, aprovechando, en la mayor medida posible, los factores naturales que limitan la propagación de dichos organismos (Organización de las Naciones para la Alimentación y la Agricultura, FAO, 2009).

Con la realización de esta investigación se pretende lograr recopilar información sobre el uso de plaguicidas botánicos en Nicaragua y revisar la eficacia del uso de los plaguicidas botánicos en los cultivos de Nicaragua y que el documento sirva como una alternativa al uso indiscriminado de los pesticidas químicos.

\section{Material y metodología}

\section{Diseño metodológico}

Esta investigación es cualitativa no experimental, se fundamenta en el método descriptivo, forma de investigación aplicada en donde no hay manipulación de variables (UNA, 2009), analizando la situación determinada con una riqueza interpretativa, contextualizando el ambiente, revisión bibliográfica y documental, apoyadas en fuentes primarias, secundarias y otras (Sampieri, Fernández, Baptista, 2003). 


\section{Análisis de contenido del estudio y delimitación del estudio}

Se realizaron revisiones bibliográficas concerniente a los estudios y trabajos escritos que se han presentado sobre los insecticidas botánicos utilizados en Nicaragua a partir del año 2000 hasta la fecha, con un análisis de los principales insecticidas botánicos registrados y evaluados en Nicaragua, tomando como base las pruebas de efectividad que se utilizaron para registrar los productos en el Instituto de Protección y Sanidad Agropecuaria.

\section{Registro y análisis de datos}

Para la elaboración del marco teórico se utilizó el mecanismo de recolección y registro de información, usando fuentes de información primaria (libros, revistas científicas, periódicos, diarios, documentos oficiales de instituciones públicas, informes técnicos e investigación de instituciones públicas o privadas), a fin de analizar antecedentes y resultados. Como fuentes secundarias se utilizaron enciclopedias, directorios, libros o artículos que interpretan otros trabajos o investigaciones sobre el uso de los insecticidas botánicos en Nicaragua.

\section{Desarrollo}

\section{Historia de los insecticidas botánicos}

El hombre depende del consumo directo de las plantas tanto vegetales, y cereales, así como de la obtención de sus productos. Anualmente, una tercera parte de la producción de alimentos se ve destruida por pestes de cultivos y productos almacenados (Ben, 2001), por lo que se hace imprescindible el estudio de nuevas vías de control de plagas. Las plantas en conjunto producen más de 100 ooo sustancias de bajo peso molecular conocidas como metabolitos secundarios, estos son, normalmente, no-esenciales para el proceso metabólico básico de la planta.

Entre ellos se encuentran terpenos, lignanos, alcaloides, azúcares, esteroides, ácidos grasos, etc. Semejante diversidad química es consecuencia del proceso evolutivo que ha llevado a la selección de especies con mejores defensas contra el ataque microbiano, o la predación de insectos y animales (Ben, 2001). Estos metabolitos secundarios tienen un rol importante en el mecanismo defensivo de las plantas, por lo tanto, en los últimos años se está retornando al uso de las plantas como fuente de pesticidas más seguros para el medio ambiente y la salud humana. Esto pesticidas pueden ser clasificados de acuerdo con el tipo de organismo frente a los cuales son eficaces: funguicidas, herbicidas, insecticidas, moluscicidas, nematicidas, rodenticidas.

\section{Problemática de los insecticidas botánicos}

Entre los agricultores circulan infinidad de recetas sobre insecticidas botánicos, usando diversas partes de la planta y mezclas de plantas (Instituto para la economía Social, IPES, 2010). Esto dificulta determinar cuál de las plantas tiene la propiedad de controlar a determinada plaga, cuáles son los ingredientes activos, las dosis empleadas, el modo de acción de cada componente y su efecto (repelente, insecticida o atrayente). Además, que la efectividad de la mayoría de los productos botánicos no ha sido en su totalidad comprobados.

\section{Generalidades de los insecticidas botánicos}

Los insecticidas botánicos tienen la propiedad de contribuir a aminorar los costos de producción de los agricultores debido a que son productos no persistentes, que confieren la más baja posibilidad de resistencia a las plagas por ser específicos, no tóxicos a animales de sangre caliente, a organismos benéficos, ni al hombre, y además se biodegradan rápidamente, no contaminan el ambiente y su costo es bajo (IPES/FAO 2010). 


\section{Tipos de compuestos naturales con actividad plaguicida}

Según Cutler et al. (1986), la interacción planta-plaga puede estar condicionada por los metabolitos secundarios de las plantas, de ahí que los compuestos naturales pueden tener actividad insecticida, nematicida, viricida, fungicida, bactericida o herbicida. El primer tipo de compuestos, se clasifican en tres grupos:

a Compuestos vegetales miméticos o antagonistas de hormonas de insectos. Camps (1988) señalo que dentro de las sustancias de este tipo de compuestos se agrupan los fitojuvenoides, antihormonas juveniles, fitoecdisteroides y antiecdisonas. Los fitojuvenoides son sustancias que inhiben la metamorfosis, las antihormonas juveniles causan una metamorfosis precoz de los insectos, los fitoecdisteroides son compuestos que inducen la muda en los insectos provocando malformaciones, esterilidad y hasta la muerte y las antiecdisonas son aquellas que interfieren alguna de las fases del proceso de la muda en los insectos.

b. Inhibidores de la alimentación. Coll (1988) planteo que las sustancias anti alimentarias se clasifican sobre la base del tipo de estructura química como terpenos, compuestos heterocíclicos, compuestos aromáticos y esteroides. Todas estas sustancias tienen como función evitar o interrumpir el proceso de alimentación del insecto (tras un consumo inicial) y conducen de esa manera a su muerte por inanición.

c. Compuestos foto tóxicos. Dentro de estos compuestos están las fototoxinas lineales, la fototoxinas cíclicas y las estructuras combinadas lineales y cíclicas.

\section{Modo de acción de los insecticidas de origen botánico}

Según Anón (1991) los bioinsecticidas obtenidos a partir de las plantas poseen varias formas de actuar, entre las que se agrupan como las más importantes:

1. Repelentes: Sustancias desagradables que contienen algunas plantas, las cuales son capaces de alejar las plagas.

2. Fagorepelentes o antialimentarios: Sustancias que interrumpen el proceso de alimentación de los insectos, incluso después de haber comenzado, poseen la propiedad de reducir la capacidad de alimentación hasta que la plaga muere por inanición.

3. Venenos por contacto: Sustancias que provocan la muerte a los insectos al ponerse en contacto con estos, por lo que para que sean efectivas tienen que aplicarse sobre la plaga.

4. Venenos estomacales: Sustancias con efecto tóxico sobre el sistema digestivo de las plagas, cuya efectividad depende de que el insecto las ingiera.

5. Acción de disfrazar olores: Este modo de acción aprovecha los olores fuertes y desagradables que expelen algunas plantas para ocultar el olor del cultivo principal y evitar que sea atacado por las plagas.

6. Uso del jabón mezclado con los insecticidas naturales: Al añadir el jabón aumenta la potencia del insecticida de dos formas; primero contribuye a deshacer la piel de los insectos con cuerpos blandos (áfidos, moscas blancas y algunos gusanos) y a su vez obstruye los espiráculos y no los deja respirar y segundo sirve como adherente, haciendo que el insecticida se adhiera mejor a las hojas del cultivo. 


\section{Familias de plantas con propiedades insecticidas usadas en Nicaragua}

Meliaceae: contiene varias sustancias activas, entre ellas azadirachtin, salanin, melantriol, las que se encuentran con mayor concentración en la semilla. Estas sustancias tienen acción de insecticida repelente, efecto anti alimentario, inhibidor de crecimiento y ovoposición, esterilizante, además acción fungicida y nematicida (Jiménez-Martínez y Varela, 2013).

Asteraceae: su principal sustancia activa es la piretrina, el piretro es un insecticida de contacto obtenido de sus flores. Los componentes de esta planta con actividad insecticida son seis esteres formados por la combinación de ácidos crisantemicos y pirétricos además los alcoholes piretrolona, cinerolona y jasmolona. El modo de acción consiste en que cuando los compuestos penetran la cutícula del insecto actúan sobre el sistema nervioso central y el sistema nervioso periférico (Jiménez-Martínez y Varela, 2013).

Solanácea: contiene una sustancia de pungencia elevada llamada capsicina, al ser aplicada sobre los insectos genera una sensación de ardor en todo el cuerpo, los insectos dejan de alimentarse, huyen del lugar y mueren (Jiménez-Martínez y Varela, 2013).

Fabaceae: es un insecticida y abono foliar que contiene flavonoides, su toxicidad se debe a la conversión por las bacterias de cumarinas a dicoumerol durante la fermentación (Lanuza y Rizo, 2012). Es un insecticida de contacto e ingestión, además actúa como repelente (Jiménez-Martínez y Varela, 2013).

Myrtáceas: El principal componente del aceite esencial es el éter óxido terpénicocineol o eucaliptol, constituyendo el 70 u $80 \%$, contiene hidrocarburos monoterpénicos, hidrocarburos sesquiterpénicos, alcoholes monoterpénicos, alcoholes sesquiterpénico y aldehídos alifáticos. El eucalipto es un insecticida de contacto e ingestión y actúa como repelente (Aguirre y Delgado. 2010).

Amaryllidaceae: contiene compuestos de azufre (tiosulfatos) los que sobre excitan el sistema nervioso de insectos y ácaros produciendo irritación, desorientación y repelencia (Jiménez-Martínez y Varela, 2013).

\section{Preparación de insecticidas naturales a base de plantas}

Se pueden utilizar las hojas, raíces, corteza y tallos, también las cabezuelas de la flor, frutos, ramas, ápices, inflorescencias, resinas y la planta completa (Jiménez-Martínez, 2015). Un aspecto de vital importancia al preparar los bioproductos lo constituye la forma de conservar al máximo los principios activos de las plantas, deben secarse en lugares aireados y colocarse en bolsas de tela, papel o cartón para que haya una mejor transpiración y puedan ser procesadas con ulterioridad sin perder su calidad (IPES/FAO 2010). Jiménez-Martínez (2015) menciona los métodos para preparar insecticidas naturales:

1. Purín: consiste en colocar las partes verdes de la planta en un recipiente lleno de agua de lluvia, posteriormente se tapa y se remueve todos los días. Después de una o dos semanas, cuando el líquido no forma espuma, se aplica diluido cerca de las raíces.

2. Infusión: se vierte agua hirviendo sobre las partes de las plantas y se deja reposar durante 24 horas.

3. Decocción: se maceran las plantas o sus partes, después de 24 horas se hierven durante 20 minutos y se dejan enfriar con el recipiente tapado.

4. Maceración: se introducen las plantas en agua (máximo tres días) evitando que se fermenten. Se filtra el líquido resultante para separarlo del material vegetal.

5. Extracto de flores: se mojan y trituran las flores, la mezcla obtenida se coloca en un lienzo fino y se presiona para extraer el líquido. El extracto debe conservarse en botellas cerradas herméticamente. 
6. Baño de semillas: se dejan caer unas gotas del extracto de la planta en un litro de agua y se mezclan, luego de 24 horas las semillas se colocan en una solución durante 10 a 15 minutos. Posteriormente se dejan secar y se siembran.

\section{Insecticidas botánicos registrados en el Instituto de Protección y Sanidad Agropecuaria}

1. Bio die: Es un producto a base de extracto de berberina, resina, argemonia y terthienyl. Tiene uso insecticida y acaricida que actúa por contacto e ingestión, no es sistémico, por lo que se recomienda una buena cobertura para lograr la máxima eficacia del producto en campo, este producto se registró en el año 2012. Este producto ha sido utilizado en hortalizas para el control de trips, palomilla dorso de diamante, gusano falso medidor y mosca blanca. Puede ser utilizado en floración, polinización, fructificación y cortes.

2. Bralic: Es un concentrado emulsionable de alta solubilidad a base de extracto de ajo, indicado como repelente de plagas agrícolas contra chupadores y aradores, posee acción disuasiva en los hábitos alimenticios de insectos plagas y obstruye la acción de las feromonas naturales causando desorientación a los insectos en su etapa de reproducción.

3. Biocontrol limoneno: Es un insecticida a base de extracto de cascara de limón, es de uso agronómico y también en poscosecha para el lavado de frutas y vegetales, actúa rápidamente por contacto destruyendo la membrana celular de los insectos en su estado de ninfa y adultos.

4. Biocontrol neem: Es un insecticida a base de extracto de neem, actúa como regulador del crecimiento, perjudicando los estados inmaduros, lo que bloquea el ciclo de mudas y provoca la muerte de insectos en estados inmaduros.

5. Insecta Pro: Es un insecticida a base de una mezcla de aceites de cedro, canela, soja, jojoba y cymbopogo, ha sido utilizado en cultivos de hortalizas, frutales y granos para el control de insectos voladores, rastreros, escarabajos, hormigas, zompopos, áfidos, ácaros, larvas y huevos, este producto se registró en el año 2017.

6. Biocan 75 CE: Es un extracto botánico elaborado a base de canela, actúa como insecticida, acaricida, el cual ejerce una acción toxica y repelente contra insectos y ácaros que atacan los cultivos. Tiene una acción toxica que provoca irritación en la epidermis de los insectos provocando la muerte en pocos minutos.

7. Nemafin: Es un extracto elaborado a base de las hojas y tallos de la planta conocida como melón amargo, actúa destruyendo la membrana de los insectos y nematodos, al momento de la aplicación provoca una inmovilización y posteriormente la muerte del insecto. Se ha utilizado para controlar plagas de hortalizas, raíces, tubérculos, frutales, granos básicos y okra.

8. Pírex: Elaborado a base de extractos de Chrysanthemum, es una piretrina natural de contacto que actúa destruyendo la membrana celular de los insectos, ninfas y ácaros de piel blanda, debe aplicarse directamente sobre el órgano de la planta que ataca la plaga.

9. Kanelite 30 SL: Elaborado a base de extractos de canela, es insecticida, acaricida y fungicida que actúa por contacto causando excitación del sistema nervioso provocando un enmascaramiento de las feromonas involucradas en el proceso de apareamiento de los insectos.

10. Quazinol 75 SL: Elaborado a base de extracto de Quassia amara, es preventivo curativo, actúa por contacto e ingesta, detiene el desarrollo de los insectos y provoca su rechazo. Se utiliza en hortalizas, raíces, tubérculos, frutales, granos básicos y okra, para el control de ácaros, afidos, pulgones, chinches, minadores, cochinillas y salta hojas.

11. Allium 98 SL: Elaborado a base de extracto de ajo, es un repelente de acción sistémico de alto espectro que es absorbido por el sistema vascular de la planta, actúa por ingesta causando trastornos digestivos.

12. Bio-Insect 8o SL: Elaborado a base de aceites esenciales que provienen del extracto de naranja, actúa por contacto destruyendo la membrana celular de los insectos, ninfas y ácaros de piel blanda.

\section{Análisis de los principales insecticidas botánicos evaluados en Nicaragua}

Evaluación de productos botánicos y biológicos para el control de plagas defoliadoras en el cultivo del repollo (Brassica Oleracea, L.)3 ${ }^{3}$ l objetivo fue evaluar la efectividad de insecticidas botánicos, biológicos

\footnotetext{
3 Carlos Gómez Calderón (1990)
} 
y un hormonal contra plagas defoliadoras del cultivo del repollo. Los tratamientos evaluados fueron: Extracto acuoso de semillas de Neem, con dosis de dos libras por bomba de 20 litros de agua. Extracto acuoso de semillas de mamey, con dosis de dos libras por bomba de 20 litros de agua. Bacillus thuringiensis I, a base de tres cepas (Nacional, Dipel y Javelin) y Júpiter, producto hormonal. Para decidir el momento de aplicación se realizaron muestreos semanales a partir de los $8 \mathrm{ddt}$, hasta la cosecha, tomando como nivel crítico o.2 larvas por planta. Los insectos encontrados en todo el ciclo del ensayo fueron Plutella xylostella L. Spodoptera sp, arañas y Polybia sp., el único insecto que llegó a nivel crítico fue P. xylostella L, apareciendo en la última semana del crecimiento vegetativo del cultivo, incrementando sus poblaciones en la etapa de formación y llenado de cabeza. Al realizar las comparaciones de las fluctuaciones poblacionales de P. xylostella L., se comprobó que el insecticida de extracto acuoso de semillas de neem es el que mejor se comporta, reportando bajas poblaciones plagas.

Evaluación de productos botánicos y químicos para el control de mosca blanca (Bemisia tabaci, Gennadius.) y geminivirus en el cultivo de tomate (Solanum esculentum, Mill.), en Tisma, Masaya. ${ }^{4}$ El objetivo fue evaluar cinco productos botánicos y químicos para el manejo del complejo mosca blanca Geminivirus en tomate, los tratamientos evaluados fueron 1) Engeo (Thiametoxam $25 \%$ y lambdacihalotrina $10.6 \%$ ) con dosis de 10 $\mathrm{cm} 3$ por bombada de 20 litros de agua. 2) Imidacloprid ${ }^{\circledR}$, formulado a base de imidacloprid, materia activa perteneciente al grupo químico de los cloronicotinilos, se aplicó dosis de $21 \mathrm{~g}$ por bombada de capacidad de 20 litros. 3) Crisantemo (Chrysanthemum cinerariaefolium Trevir), dosis de $450 \mathrm{~g}$ de producto molido, lo que incluye hojas y flores frescas por bomba de capacidad de 20 litros, 4) Madero negro (Gliricidia sepium Jacq), con dosis de $450 \mathrm{~g}$ de producto macerado (hojas frescas) por bomba de 20 litros. 5) Chile + Ajo + Jabón, la dosis utilizada fue de 4 oz de chile molido, 4 oz de ajo molidas más 2 oz de jabón por bombada de 20 litros. 6) Testigo, consistió en la aplicación de agua una vez a la semana. Las poblaciones de moscas blancas se presentaron a partir de la primera fecha de muestreo, ( 25 de noviembre) en todos los tratamientos. El Crisantemo presento los menores promedios de mosca blanca con respecto a los demás, seguido de los tratamientos Chile+Ajo+Jabón y Madero Negro.

Insecticidas botánicos en el manejo del chinche patas de hoja (Leptoglossus zonatus, Dallas, Hemiptera: Coreidae) y la mosquita negra (Trigona silvestrianun, Vachall, Himenoptera: Apidae) y su efecto sobre los enemigos naturales en el cultivo de marañón (Anacardium occidentale L.), León, Nicaragua. ${ }^{5}$ El objetivo fue evaluar cuatro alternativas botánicas para el manejo del chinche patas de hoja y la mosquita negra. Las alternativas botánicas fueron 1) Chile + ajo + jabón, 2) cebolla + crisantemo, 3) chile + ajo, 4) chile + jabón, con dosis de 11 de solución por bomba de 201 para todos los tratamientos. Las variables evaluadas fueron número de chinches y mosquitas negras por árboles. Se comparó la fluctuación poblacional del chinche patas de hoja y se observó que las poblaciones de este insecto variaron en todos los tratamientos evaluados, encontrándose los mejores resultados utilizando chile + ajo y chile + ajo + jabón con 0.75 chinches por árbol para ambos tratamientos. Se observaron las fluctuaciones poblaciones de mosquita negra en donde se presentaron desde la primera fecha de muestreo (febrero 11), hasta la última (mayo o8), encontrando las poblaciones más bajas con chile + ajo + jabón seguido de chile + jabón con 1 y 2 insecto por árbol respectivamente. Los tratamientos que presentaron las poblaciones más altas fueron el testigo y cebolla + crisantemo con 22 y 18.5. Se observó poca afectación sobre las poblaciones de organismos benéficos asociadas al cultivo del marañón. Los mayores rendimientos fueron en las plantas tratadas con chile + ajo y chile + ajo + jabón con mayores brotes florales, se considera la efectividad de estos extractos para el manejo de chinches y mosquita negra.

Uso de plaguicidas químicos y botánicos para el manejo de las principales plagas de la chiltoma (Capsicum annum L.) en Tisma, Masaya. ${ }^{6}$ El objetivo fue evaluar los diferentes productos químicos y botánicos para el manejo del acaro blanco y otros insectos plagas claves en el cultivo de la chiltoma. Los insecticidas evaluados fueron 1) Oberón, con el ingrediente activo Spiromesifen y dosis de $7.4 \mathrm{ml}$ por bombada de 20 litros, 2) chile + ajo + jabón con dosis de $200 \mathrm{~g}$ de chile indio molido, dos cabezas de ajo ( $20 \mathrm{~g}$ ) molido ambos en un

\footnotetext{
4 Eliezer Lanuza Rodríguez y Edwin Rizo Gonzales (2010).

5 Jorge Gómez Martínez (2009-2010).

6 Javier Barberena y Yasmir Lacayo (2009).
} 
litro de agua y $35 \mathrm{~g}$ de detergente por bombada de 20 litros, 3) Rienda, insecticida y acaricida (deltametrina y triazofos) con dosis de $7.4 \mathrm{ml}$ por bombada de 20 litros, 4) Vertimec, acaricida insecticida (Abamectina) con dosis de $18 \mathrm{ml}$ por bombada de 20 litros, 5) Sulfato de amonio, se aplicó de forma foliar con dosis de 50 g por bombadas de 20 litros y un 6) testigo, aplicación de agua. El insecticida chile + ajo + jabón, controló la plaga, pero no con la eficiencia que se esperaba, se encontraron altas poblaciones de insectos benéficos, mientras que en las parcelas donde se le aplico insecticidas químicos las poblaciones de insectos benéficos fueron muy bajas, por lo que se recomienda considerar las alternativas de este insecticida botánico, tomando en cuenta que no presentan la misma persistencia que los químicos.

Plaguicidas botánicos y químicos para el control del ácaro blanco (Polyphagotarsonemus latus Bank) (Acarina: tarsonemidae) en chiltoma (Capsicum annuum L.), Tisma, Masaya. ${ }^{7}$ El objetivo fue evaluar productos botánicos y químicos para el manejo del acaro blanco en el cultivo de la chiltoma. Los tratamientos evaluados fueron: Chile + Jabón con dosis de $100 \mathrm{~g}$ de chile molido (Chile Congo, cascara y semillas) en 1 litro de agua más $1 / 4$ de jabón. Oberón con dosis de $7.4 \mathrm{ml}$, neem con dosis de $35 \mathrm{~g}$ de producto molido. Se utilizó Vertimec con dosis de $18 \mathrm{ml}$, ajo + jabón, utilizando dos cabezas de ajo molidas más 1/4 de jabón, y un testigo (agua). Las aplicaciones se realizaron por las tardes y por aspersión directa al follaje haciendo uso de una bomba de mochila con capacidad de 20 litros. Las poblaciones del ácaro blanco presentaron dos picos poblacionales a los 49 ddt y 77 ddt, los tratamientos botánicos Chile + Jabón presento los mejores resultados con 14.66 ácaros blancos por planta.

Productos botánicos para el manejo del ácaro blanco (Polyphagotarsonemus latus, Banks.) (Acarina; Tarsonemidae), en chiltoma (Capsicum annuum L.), en Masaya, Nicaragua. ${ }^{8}$ El objetivo es evaluar diferentes productos botánicos para el manejo del acaro blanco en el cultivo de la chiltoma. Los tratamientos evaluados fueron, 1) Eucalipto con dosis de $454 \mathrm{~g}$ de hojas de eucalipto molido, disuelto en un litro de agua, con 24 horas de reposo, 2) neem, dosis de $10 \mathrm{ml}$ por litro de agua, 3) crisantemo con dosis de 908 gramos de flores y tallos molidos y disueltos en un litro de agua, con 24 horas de reposo, 4) madero negro con dosis de $454 \mathrm{~g}$ de hojas molidas y disueltas en un litro de agua, con 24 horas de reposo, 5) chile + Ajo + Jabón, se utilizaron 100 gramos de chile, 28 gramos de detergente y una cabeza de ajo, molido y disuelto en un litro de agua, con 24 horas de reposo, y un tratamiento testigo (agua), todos los tratamientos se aplicaron en bombas con capacidad de 20 litros de agua. Las poblaciones del ácaro blanco se presentaron a partir de la primera fecha de muestreo y se observó que las poblaciones de este insecto variaron en todos los tratamientos evaluados, se encontró que los tratamientos que ejercen el mejor efecto de control del ácaro blanco y el rendimiento son madero negro seguido de chile+ajo+jabon.

Evaluación de extractos botánicos para el manejo de insectos plagas asociados al cultivo del pipián (Cucurbita pepo L.), en El Plantel, Masaya. ${ }^{9}$ El objetivo fue evaluar cuatro extractos botánicos para el manejo de plagas asociadas al cultivo del pipián. Las alternativas evaluadas fueron: 1) Chile+ajo+detergente, dosis de 1 1, 2) chile+ detergente, dosis de 11,3 ) madero negro, dosis de 1 1, 4) neem, dosis de 1 1, 5) testigo absoluto, se aplicó solamente agua. Para determinar el momento de aplicación de cada tratamiento se realizaron muestreos semanalmente utilizando la metodología de Martínez y Jiron (2009) considerando las plagas principales (Mosca blanca, minador, maya, Diabrotica sp. y pulgones), se utilizó una bomba de mochila con capacidad de 20 litros de agua en todos los tratamientos. Los tratamientos neem y chile+ajo+detergente obtuvieron mejor efecto en el manejo de pulgones, maya y tortuguilla, madero negro y chile+ajo+ detergente obtuvieron mejor efecto sobre minador, mosca blanca y menos efecto en organismos benéficos como lorito verde, avispa y Solenopsis sp., mientras que neem y chile+ajo+ detergente tuvieron efectos negativos sobre estos insectos.

Evaluación de insecticidas químicos, biológicos y botánicos para el manejo del pulgón amarillo (Melanaphys sacchari, Zehnter), y otras plagas e insectos benéficos en sorgo (Sorghum bicolor L. Moench), en finca el Plantel,

\footnotetext{
7 Javier Barberena y Yasmir Lacayo (2009).

8 Ilda Rayo Cruz y Ariel Mena García (2014).

9 Junior Borst Leiva y Malkin Rodríguez González (2017)
} 
Masaya.10 El fue objetivo evaluar productos químicos, biológicos y botánicos para el manejo del pulgón amarillo en el cultivo del sorgo. Las alternativas evaluadas fueron: Engeo, Imidacloprid, Beauveria bassiana, Metharizium anisopliae y chile+ajo+jabón en comparación con un testigo que solamente se le aplico agua, para determinar el momento de aplicación de cada uno de los tratamientos se utilizó la metodología propuesta por Martínez y Jirón (2009). Las poblaciones de insectos se encontraron en todas las fechas de muestreos, chile+ajo+jabón presento como promedio 22.5 pulgones por planta, este mismo tratamiento y los insecticidas biológicos tuvieron menor efecto sobre mariquita, arañas y huevos de león de áfidos, mientras que los químicos afectaron las poblaciones de benéficos.

\section{Conclusiones}

Los insecticidas botánicos son una alternativa dentro del manejo integrado de plagas, tienen la propiedad de aminorar los costos de producción por ser productos no persistentes, confieren la más baja resistencia a plagas, no ocasionan daños a organismos benéficos y se biodegradan rápidamente.

En Nicaragua se han registrado 12 insecticidas botánicos desde el 2011 hasta el año 2017, todos estos a base de extractos acuoso de planta, semillas, hojas y flores que se han utilizado en hortalizas, frutales, okra, raíces y tubérculos, para el control de plagas como mosca blanca, afidos, ácaros y trips.

Actualmente en Nicaragua se han realizado ocho en el periodo entre mayo 1990 y noviembre 2017 sobre el uso y manejo de insecticidas botánicos a base de extractos de chile, ajo, crisantemo, madero negro y neem en los cultivos de tomate, chiltoma, repollo, sorgo y marañón con el propósito de controlar las principales plagas que atacan a estos cultivos.

\section{Agradecimiento}

Esta publicación obtuvo el financiamiento de: El Fondo de Asistencia Internacional de los Estudiantes y Académicos Noruegos (SAIH).

\section{Lista de referencias}

Aguirre, V., y Delgado, V. (2010). Pesticidas naturales y sintéticos. Revista Ciencia,13(1), 43-53.

Alonso, O. (1998). Los insecticidas botánicos: una opción ecológica para el control de plagas. Estación Experimental de Pastos y Forrajes "Indio Hatuey" Matanzas, Cuba. http://payfo.ihatuey.cu/Revista/ v22n1/pdf/pyfo119g.pdf

Ben, J. (2001). Antifeedant activity of plant extracts and of new natural diglyceride compounds isolated from Ajuga pseudoiva leaves against Spodoptera littoralis larvae. http://uaim.edu.mx/webraximhai/ Ej-25barticulosPDF

Camp. (1988). Insecticidas biorracionales. Escrito por Consejo Superior de Investigaciones Científicas de España. $\quad$ https://books.google.com.ni/books?hl=es\&lr=\&id=43izwogjzugc\&oi=fnd\&pg $=$ pa1\&dq=insecticidas+biorracionales+camps+1988\&ots=8txwmow55i\&sig=ddvizxrutjpvhjqc574racqdtuk\#v=onepage\&q=insecticidas\%20biorracionales\%20camps\% 20 $\underline{1988 \& \mathrm{ff}=\text { false }}$

Carrero, J. (1996). Lucha Integrada contra las Plagas Agrícolas y Forestales. Madrid, España: Mundi Prensa.

Cutler., Severson., y Cole. (1986). The plant-pest interaction may be conditioned by the secondary metabolites of the plants. $n$ book: Natural Resistance of Plants to Pests: Roles of Allelochemicals. https://www.

$\overline{10}$ por Nelson Reyes Canales y Lenard Rivas (2017). 
researchgate.net/publication/265510117 Secondary metabolites from higher plants Their possible role as biological control agents

Organización de las Naciones Unidas para la alimentación y Agricultura. (2009). Definición de Manejo Integrado de Plagas (MIP). Biología de Entomógagos Verano. Recuperado de http://www.cm.colpos.mx/ moodle/file.php/10/U Conceptos MIP e inclusion de EN.pdf

Gómez, C. (1990). Evaluación de productos botánicos y biológicos para el control de plagas defoliadoras en el cultivo del repollo (brassica oleracea). (Tesis de grado). Universidad Nacional Agraria. Managua, Nicaragua.

Instituto para la Economía Social. (2010). Biopreparados para el manejo sostenible de plagas y enfermedades en la agricultura urbana y periurbana. Santiago, chile.

Jiménez-Martínez, E. S. (2009). Manejo integrado de plagas. Managua, Nicaragua: Universidad Nacional Agraria.

Jiménez-Martínez, E. S. (2015). Preparación de bioplaguicidas para el manejo de plagas y enfermedades. Managua, Nicaragua: Universidad Nacional Agraria.

Jiménez-Martínez, E. S., y Varela, G. (2013). Módulo Práctico: Manejo Integrado de Plagas. Managua, Nicaragua: Universidad Nacional Agraria.

Lanuza, E., y Rizo, E. (2012). Evaluación de productos botánicos y químicos sobre el complejo mosca blanca (Bemisia tabaci Gennadius)- Geminivirus en el cultivo de tomate (Solanum esculentum, Mill.), en TismaMasaya. (Tesis de grado). Universidad Nacional Agraria. Managua, Nicaragua.

Martínez, R., y Jirón, M. (2009). Evaluación de productos botánicos y químicos para el manejo del ácaro blanco (Poliphagotarsonemus latus, Bank.) y otras plagas claves en el cultivo de chiltoma (Capsicum annuum L.) y su efecto en los enemigos naturales en Tisma, Masaya. (Tesis de Gardo). Universidad Nacional Agraria. Managua, Nicaragua.

Sevilla, E., y Rodríguez, E. (2009). Evaluación de alternativas químicas y botánicas para el manejo del acaro blanco (Poliphagotarsonemus latus, Bank) en chiltoma (Capsicum annuum L.), Tisma, Masaya. (Tesis de grado). Universidad Nacional Agraria. Managua, Nicaragua.

Rayo, I., y Mena, A. (2015). Evaluación de cinco productos botánicos para el manejo del ácaro blanco (Polyphagotarsonemus latus, Banks.) en chiltoma (Capsicum annuum L.), en Tisma, Masaya. (Tesis de grado). Universidad Nacional Agraria. Managua, Nicaragua.

Reyes, N., y Rivas, L. (2017). Evaluación de insecticidas químico, biológico y botánico para el manejo del pulgón amarillo (Melanaphis sacchari, Zehnter), y otras plagas e insectos benéficos, en sorgo (Sorghum bicolor L. Moench), en "El Plantel" 2017. (Tesis de grado). Universidad Nacional Agraria. Managua, Nicaragua.

Rodríguez, H. C. (2000). Plantas contra plagas: Potencial de ajo, anona, nin, chile y tabaco. Red de acción sobre plaguicidas y alternativas en México. Ciudad de México, México.

Sampieri, R., Fernández, C., y Baptista, P. (2003). Metodología de la Investigación. Ciudad de México, México: McGraw Hill Interamericana.

Singh, A., Singh, D. K., Mishra, T. N., y Agarwal, R. A. (1996). Molluscicides of plant origin. Biol. http://www. scielo.br/scielo.php?script=sci arttext\&pid=S0100879X1998000700011 
Universidad Nacional Agraria. (2008). Guías y Formas Metodológicas de la Forma de Culminación de estudios. Managua, Nicaragua: UNA. 